\title{
VIOLENCIA, DOMINANCIA Y MANIPULACIÓN: UN ANÁLISIS CRIMINOLÓGICO-INTEGRADOR
}

\author{
Maximiliano Hernández Cuevas*
}

\begin{abstract}
Resumen: Este trabajo utiliza un enfoque integrador para examinar los comportamientos violentos, dominantes y manipuladores, así como sus motivaciones. Tras exponer estudios guiados por paradigmas de investigación distintos, pero complementarios sobre los rasgos de personalidad malevolentes, demuestra que es erróneo basarse en el criterio de la adaptación social para establecer la normalidad y salud mental de los individuos. Esto, porque muchos personajes perfectamente adaptados y hasta con posiciones de liderazgo en múltiples esferas de la vida en sociedad incurren en comportamientos profundamente nocivos y antisociales, lo cual hace necesario modificar nuestros referentes científicos de la normalidad o salud del comportamiento humano.
\end{abstract}

Palabras clave: violencia; poder; antisocial; normalidad; salud.

* Doctor en Ciencias Políticas y Sociales con orientación en Sociología Jurídica, Universidad Nacional Autónoma de México; maestro en Ciencias Penales con Especialidad en Criminología, Instituto Nacional de Ciencias Penales; catedrático del Instituto Nacional de Ciencias Penales; profesor investigador de la Universidad Autónoma de la Ciudad de México; docente y fundador de la Licenciatura en Derecho en el Programa Universitario de Educación Superior en Centros de Reclusión de la Ciudad de México (PESCER), impartida a los reclusos; miembro del Sistema Nacional de Investigadores, Consejo Nacional de Ciencia y Tecnología.Ciudad de México.Correo-e: maximiliano.hernandez@uacm.edu.mx.Fecha de recepción: 18 de agosto de 2020. Fecha de aceptación: 12 de enero de 2021. Para citar el artículo: Maximiliano HeRnÁndez Cuevas. "Violencia, dominancia y manipulación: un análisis criminológicointegrador", Revista Derecho Penal y Criminología, vol. 41, n. ${ }^{\circ} 111$, julio-diciembre de 2020, Bogotá, Universidad Externado de Colombia, pp. 109-132. Dor: https://doi.org/10.18601/01210483.v41n111.04. 


\title{
VIOLENCE, DOMINANCE AND MANIPULATION: A CRIMINOLOGICAL-INTEGRATIVE ANALYSIS
}

\begin{abstract}
This work uses an integrative approach to examine violent, dominant, and manipulative behaviors, as well as their motivations. After exposing studies guided by different, but complementary research paradigms on malevolent personality traits, he shows that it is wrong to base oneself on the criterion of social adaptation to establish the normality and mental health of individuals. This, because many perfectly adapted characters and even with leadership positions in multiple spheres of life in society, engage in deeply harmful and antisocial behavior, what makes it necessary to modify our scientific references of the normality or health of human behavior.
\end{abstract}

Keywords: violence; power; antisocial; normality; health.

\section{INTRODUCCIÓN: ADVERTENCIA EPISTEMOLÓGICA SOBRE EL ESTUDIO DE LA INCLINACIÓN INDIVIDUAL A CAUSAR DAÑO}

La necesidad de entender los comportamientos violentos, dominantes y manipuladores, así como sus motivaciones, ha derivado en múltiples teorías basadas en paradigmas de investigación elaborados en función del asunto examinado y según las posturas asumidas sobre lo que se considera científicamente válido.

Así pues, en la historia de la ciencia ${ }^{1}$ encontramos que el conocimiento se genera a partir de fenómenos observables, objetivos y mensurables; es lo primero que la mente humana percibe y comienza a procesar reflexiva y empíricamente. De ahí, por ejemplo, que sea posible ubicar a la teoría de la verdad "por correspondencia"2 como una de las más influyentes, porque se basa en la relación entre lo que percibimos -vía nuestros sentidos- y los objetos, a saber: la realidad objetiva, esto es, basada en el estudio de los objetos y los hechos. Es una teoría sobre la verdad obtenida empíricamente.

Por su parte, más compleja de entender resulta la teoría de la verdad "por consenso"3. Esto, porque -entre otras razones- alude a conocimientos surgidos de la realidad

1 Vid. Piaget, Jean y García, Rolando. Psicogénesis e historia de la ciencia, 11. a ed., México, Siglo XXI, 2008.

2 Alfred Tarski. "Verdad y demostración", Disputatio. Philosophical Research Bulletin, vol. 4, n. ${ }^{\circ} 5$, Dic. 2015, pp. 367-396 [en línea], disponible en [www.disputatio.eu]; Tarski, Alfred. La concepción semántica de la verdad y los fundamentos de la semántica, 1966 [en línea], disponible en [http://serbal. pntic.mec.es/ cmunoz11/tarski.pdf].

3 "[La verdad por correspondencia] nos permite afirmar que 'los enunciados son verdaderos, y solo ahora verdaderos, cuando se orientan en el sentido de la realidad o la retratan totalmente, son isomorfos 
subjetiva o simbólica, es decir, no palpable "objetivamente" sino existente en el marco simbólico, más que fáctico: de las representaciones o simbolismos compartidos culturalmente. Se trata de investigar el sentido de la realidad o, dicho de otra forma, de examinarla en un nivel diferente al de los objetos y los hechos.

Tenemos, entonces, que la realidad se despliega más allá de lo que se nos presenta objetivamente; la verdad por correspondencia sólo nos muestra una porción de un entorno más complejo que precisa de la descripción, interpretación, comprensión, explicación y verificación por otros medios. Buscar la verdad por consenso implica investigar lo existente en niveles distintos de realidad, para lo cual es indispensable el uso de paradigmas de investigación alternativos y complementarios al de la llamada "ciencia positiva" (productora de verdades con base en objetos y hechos observables y medibles $)^{4}$.

Así, los conocimientos obtenidos al estudiar el sentido de la realidad resultan verificables de manera cualitativa más que cuantitativa, pues las verdades son descubiertas desde un puesto de investigación participante, en el cual el investigador no "objetiva" la realidad investigada, sino que se asume como parte de ella ${ }^{5}$. Es viable observar que la verdad por consenso atañe a la actividad transdisciplinaria ${ }^{6}$ en la cual los hallazgos

con la realidad, etc.' Pero justamente en esto consistiría su límite; ellos, de hecho, no tendrían en cuenta que 'la correspondencia entre los enunciados y la realidad debe, a su vez, ser explicitada mediante enunciados'. En definitiva, no podríamos atribuir al término 'realidad' ningún otro sentido que aquello que consideramos implícito en los enunciados verdaderos, relativos a estados de cosas existentes. No podríamos introducir el concepto 'realidad' de modo independiente al término 'enunciado verdadero'. Por tanto -y he aquí el sentido de la teoría consensual de la verdad- 'no puedo atribuir un predicado a un objeto, si y solo si, cualquier otro que pudiera entrar en coloquio conmigo atribuirla el mismo predicado al mismo objeto. Para distinguir los enunciados verdaderos de los falsos, yo me baso en el juicio de otros [...]. La condición para la verdad de los enunciados es la adhesión potencial de todo otro": Habermas, Jürgen. "Vorbereitende Bemerkungen zu einer Theorie der kommunikativen Kompetenz", en Habermas-Luhmann. Theorie der Gesellschaft oder Sozialtechnologie, Suhrkamp Verlag, Frankfurt, 1971; en Belardineli, Sergio. La teoría consensual de Jürgen Habermas, Anuario filosófico, 1991, p. 117 [en línea], disponible en [https://dadun.unav. edu/bitstream/10171/2322/1/02.\%20Sergio\%20Belardinelli.\%20La\%20Teor\%C3\%ADa\%20Consensual\%20de\%20La\%20Verdad\%20de\%20J\%C3\%BCrgen\%20Habermas.pdf].

4 Sobre los paradigmas de investigación, vid. Enríquez Rubio Hernández, Herlinda. Investigación científica en el derecho y disciplinas afines. Un proceso epistémico-metodológico riguroso, México, Porrúa, 2019, pp. 41-56; Hernández Cuevas, Maximiliano. La investigación argumentada. Bases del discurso en la ciencia y en el derecho, México, Porrúa, 2017, pp. 6-21 y 35-45; Enríquez Rubio Hernández, Herlinda y Hernández Cuevas, Maximiliano. Consideraciones epistémico-metodológicas sobre la investigación de la realidad jurídico-social, México, Instituto de Investigaciones Jurídicas de la Universidad Autónoma de Chiapas, 2014, pp. 57-70 [en línea], disponible en [www.iij-unach.mx].

5 "Comprender lo que se dice precisa participación y no mera observación": Habermas, Jürgen. Consciencia moral y acción comunicativa, Madrid, Trotta, 2008, p. 37; Habermas, Jürgen. Teoría de la acción comunicativa, México, Taurus, 2002, pp. 158-169.

6 Cf. Basarab, Nicolescu. La transdisciplinariedad. Manifiesto, Ed. Du Rocher, 1996; Morin, Edgar. El método III. El conocimiento del conocimiento, Madrid, Cátedra, 1999; Sergio Néstor Osorio García. 
y argumentos son analizables y debatibles a través de diversos - pero complementarios- enfoques; debates desde donde se "filtra lo que es racionalmente aceptable para todo el mundo" ". Así, la aceptabilidad racional de los juicios mejor sustentados de unos y otros, debatiendo en circunstancias de plena igualdad, se vuelve fundamental: "sirve como sustituto para la referencia ontológica a un mundo objetivo"

Sobre tales aserciones, en lo alusivo al estudio de los comportamientos violentos, dominantes y manipuladores, este trabajo parte de la hipótesis de que el criterio de adaptabilidad social es erróneo para establecer la normalidad y salud mental de una persona en su interacción dentro de la vida en sociedad, dado que existen múltiples individuos notoriamente encubiertos en una vida adaptada y, no pocos, con elevado reconocimiento social, cuyas acciones resultan gravemente antisociales.

De tal forma, el objetivo aquí es lograr una perspectiva integradora como la mejor opción descriptiva, de análisis, interpretación y explicación para demostrar que es erróneo el uso de la adaptación social como criterio válido de normalidad y salud mental; es decir que es necesario contar con referentes distintos con el fin de evitar encubrir la malevolencia social propia de la personalidad, el carácter y las motivaciones de los individuos proclives a causar daño (en todo lo que esto implica, desde los efectos de sus acciones nocivas en el círculo íntimo de la convivencia humana hasta en las diversas esferas de la vida social y del entorno natural).

\section{INVESTIGACIÓN DE LOS COMPORTAMIENTOS VIOLENTOS, DOMINANTES Y MANIPULADORES}

Las teorías psicológicas tienen una larga trayectoria empírica desarrollada desde hace décadas básicamente en los Estados Unidos de América, en las que se le da mucha importancia al término personalidad. Sin embargo, al explorarlas encontramos la ausencia notoria del concepto carácter, más asociado a la tradición científica de origen europeo. Esto revela dos formas básicas o tradiciones teóricas de abordar el estudio del comportamiento humano:

1.. La de origen estadounidense, que ubica a la psicología fundamentalmente como ciencia experimental y, por consiguiente, llanamente empírica; lo cual hace notorio el desarrollo de sus indagaciones, especialmente orientadas por el paradigma positivista

\footnotetext{
"El pensamiento complejo y la transdisciplinariedad: fenómenos emergentes de una nueva racionalidad", Rev. Fac. Cienc. Econ., vol. Xx, n. ${ }^{\circ}$ 1, Universidad Militar Nueva Granada, junio de 2012 , pp. 269-291.

7 Habermas, Jürgen. La ética del discurso y la cuestión de la verdad, 3. impresión, México, Paidós, 2010 , p. 84.

8 Ibídem, p. 88.
} 
de investigación, dado que su interés es describir y explicar objetivamente la personalidad y el comportamiento humano ${ }^{9}$.

2. a . Por su parte, la tradición de origen europeo, centrada más en una perspectiva psicodinámica, aborda el estudio de la personalidad y del comportamiento sobre un sustento epistemológico diferente al de la llamada "ciencia positiva". Así, se reconoce al psicoanálisis como psicología dinámica, la cual, básicamente, trabaja con la guía dada por el paradigma hermenéutico de investigación ${ }^{10}$. Ello, porque su interés se centra en la búsqueda del origen de las motivaciones profundas, su significado y orientación del carácter y la personalidad. De esta forma, ambos conceptos son ingredientes primordiales donde, por cierto, el papel del inconsciente es central para el examen del comportamiento impulsivo y racional del ser humano.

\subsection{Estudios empíricos guiados por el paradigma positivista de investigación}

En relación con la primera de las escuelas, esmerada primordialmente en la obtención de datos empíricos de la personalidad -es decir, detectables, registrables y mensurables de manera confiable-, se han producido muchas teorías: unas abocadas al descubrimiento y la perfilación de sus rasgos o características, y otras, incluso, al condicionamiento o moldeo experimental de la conducta y actitudes ante la vida ${ }^{11}$.

En particular, una de las más actuales y reconocidas es la teoría de los "cinco grandes factores o rasgos de la personalidad". Basa sus afirmaciones en el análisis factorial y tiene antecedentes en los trabajos de otra de las muy importantes teorías sobre análisis factorial de la personalidad ${ }^{12}$. Goza de gran aceptación en la comunidad científica debido tanto a sus antecedentes teóricos ${ }^{13}$ como a su sencillez y su eficaz planteamiento y desarrollo: haber analizado el lenguaje cotidiano empleado por la gente para describir la personalidad: "el lenguaje de la vida diaria debería, a lo largo de los siglos, haber llegado a reflejar dimensiones importantes de la personalidad" 14 .

9 Vid. Enríquez Rubio Hernández, Herlinda, Investigación científica en el derecho..., cit., pp. 41-43; Enríquez Rubio Hernández, Herlinda y Hernández Cuevas, Maximiliano. Consideraciones epistémicometodológicas sobre la investigación..., cit., p. 64.

10 Ibídem, p. 65.

11 Al respecto, un buen tratado sobre las teorías de la personalidad: Cloninger, Susan C. Teorías de la personalidad, 3. a ed., México, Pearson Educación, 2003.

12 La teoría de Raymond Cattell, de minucioso trabajo empírico que describe hasta 16 factores o rasgos de la personalidad, cuyo puntaje arrojado en inventarios que miden los $16 \mathrm{~F}$ (factores), en conjunto, define el perfil de personalidad de alguien: "[L]os rasgos son unidades de personalidad que tienen valor predictivo [...] definen lo que hará una persona cuando se enfrente con una situación determinada [...] existen como determinantes de la conducta”, ibídem, p. 234.

13 Ibídem, pp. 227-250.

14 Enfoque léxico, ibídem, p. 251. 
Los "cinco grandes" han sido explorados varias veces con test de personalidad aplicados a la población estadounidense. Son rasgos útiles para caracterizar y establecer diferencias entre las personas y han tenido varias denominaciones, pero en la actualidad se reconocen las siguientes: extraversión, afabilidad, rectitud (responsabilidad), neuroticismo (ansiedad) y apertura (a la experiencia, lucidez). Conviene advertir que esta teoría se interesa en describir estos rasgos, no en explicar sus orígenes. Sin embargo, otros estudios, también teórico-empíricos, sí han puesto el interés en explorar el posible origen genético o ambiental de ciertos rasgos de la personalidad no tan extendidos en la población ordinaria como son los "cinco grandes", pero que, no obstante, también tienen presencia entre los comportamientos de las personas comunes.

Estos son los abordajes acerca de la tríada, que posteriormente pasó a ser la tétrada obscura de la personalidad ${ }^{15}$. Son varios los análisis empíricos que han abordado el asunto y cabe decir que, a principios del siglo XXI, el interés prevaleciente estuvo centrado, más que en el origen, en la correlación de estos rasgos con los "cinco grandes". Así, primero se buscó, entre población estudiantil universitaria, el grupo de tres rasgos que habían sido previamente estudiados y definidos - por separado- con base en inventarios de personalidad aplicados en muestras estadounidenses de individuos normales y el Manual Diagnóstico y Estadístico de los Trastornos Mentales de la Asociación Americana de Psiquiatría (DSM) ${ }^{16}$.

El punto clave del estudio estuvo en que esos rasgos fueron reconocidos y examinados como tríada: el maquiavelismo, el narcisismo y la psicopatía; los dos últimos, subclínicos (entendiéndose por "subclínico" la característica de "no patológicos"; es decir, que el nivel de anomalía no es tan pronunciado como el correspondiente a los trastornos de la personalidad del grupo B del DSM $)^{17}$.

El énfasis del estudio fue contrastar la tríada con los "cinco grandes" para detectar diferencias y similitudes, y encontró: (a) sólo una correlación común con los "cinco": en la baja afabilidad; (b) que la sustancial sobreestima propia de los narcisistas podría también ser evidente en el maquiavelismo y la psicopatía subclínica; (c) que

15 Paulhus, Delroy L. y Kevin Williams fueron los primeros en integrar la tríada y, posteriormente, introducir al sadismo como un cuarto rasgo con que formar la tétrada. Sobre ésta, vid. Delroy L. Paulhus. "Toward a Taxonomy of Dark Personalities", Current Directions in Psychological Science, vol. 23 (6), 2014, pp. 421-426 [en línea], disponible en [cdp.sagepub.com].

16 Paulhus, Delroy L. y Kevin Williams. "The Dark Triad of Personality: Narcissism, Machiavellianism and Psychopathy", Journal of Research in Personality 36, usA, Elsevier Science, 2002, pp. 556-563.

17 Grupo B o de trastornos de la personalidad, integrado por los siguientes trastornos clínicos: trastorno antisocial de la personalidad, trastorno de la personalidad límite, trastorno de la personalidad histriónica y trastorno de la personalidad narcisista, vid. American Psychiatric Association. Guía de consulta de los criterios diagnósticos del DSM-V, Chicago, American Psychiatric Publishing, A division of American Psychiatric Association, Washington, D. C.-London, England, Traducción: Burg Translations Inc., 2014, pp. 363-366. 
estos dos últimos están negativamente correlacionados con el rasgo rectitud de los "cinco grandes"; (d) únicamente los psicópatas puntearon bajo en neuroticismo, lo cual coincide con que generalmente se les caracteriza como carentes de ansiedad; (e) los narcisistas muestran la mayor sobreestimación de sí mismos, seguidos por los psicópatas; aunque ello es por un fuerte ingrediente de autoengaño, es decir, que tienen poca perspicacia sobre su personalidad; (f) la sobreestima de sí mismos de los psicópatas resultó ser novedosa según este estudio; (g) en cambio, el maquiavelismo no reveló signos de sobreestima propia, sino mayor ubicación en la realidad respecto a su autoimagen; (h) tanto el narcisismo como la psicopatía mostraron contrastes de grandiosidad y poca perspicacia sobre sí mismos; (i) la psicopatía, al carecer de ansiedad, muestra ser -entre la tríada- el rasgo más impregnado de capacidad de traición; (j) el maquiavelismo y la psicopatía revelaron correlación alta en los niveles de inteligencia no verbal; $(\mathrm{k})$ una gran variedad de mediciones predijeron alto comportamiento antisocial sólo en la psicopatía, no así para el maquiavelismo y el narcisismo; (1) la conclusión final del estudio es que esta tríada no es normal en la población común, pero existe entre la misma en un nivel considerado subclínico o "no patológico"18.

En síntesis, el estudio revela que la tríada comparte, en diverso grado, características propias de personajes socialmente malévolos con tendencias de comportamiento a la autopromoción, frialdad emocional, poca sinceridad y agresividad.

\subsubsection{Estudios del origen genético, niveles de desarrollo moral y perturbación del comportamiento malevolente}

Varias investigaciones han examinado a la tríada para: (1) indagar sobre el origen genético o ambiental de los rasgos de personalidad, correlacionando los “cinco grandes" y la tríada ${ }^{19}$; (2) considerar tal origen y el nivel de desarrollo moral de sus individuos $^{20}$; y (3) examinarla con base en el grupo B del Manual Diagnóstico y Estadístico de los Trastornos Mentales de la Asociación Americana de Psiquiatría (DSM-V) ${ }^{21}$. Veámoslas con algún acercamiento.

18 Paulhus, Delroy L. y Kevin Williams. “The Dark Triad of personality...,, cit., pp. 556-563.

19 Vernon, Philip A.; Vanessa C. Villani; Leanne C. Vickers y Julie Aitken Harris. "A behavioral genetic investigation of the Dark Triad and the Big 5", Personality and Individual Differences 44, 2008, pp. 445-452 [en línea], disponible en [https://www.journals.elsevier.com/personality-and-individualdifferences].

20 Campbell, Jennifer; Julie Aitken Schermer; Vanessa Villani C.; Brenda Nguyen; Leanne Vickers y Philip A. Vernon. "A Behavorial Genetic Study of the Dark Triad of Personality and Moral Development”, Twin Research and Human Genetics, vol. 12, n. ${ }^{\circ}$ 2, 2009, pp. 132-136.

21 Vossen, Taylor J.; Frederick L. Coolidge; Daniel L. Segal y Jennifer J. Muehlenkamp. "Exploring the Dark Side: Relationships between the Dark Triad Traits and Cluster B Personality Disorder Features", J. Psychiatry Psychiatric Disord, 1 (6), 2017, pp. 317-326. 
(1) Respecto a la indagación sobre el origen genético o ambiental de los rasgos obscuros de la personalidad, el reporte correspondiente dice haberla realizado con 278 pares de gemelos adultos a quienes se les aplicó un cuestionario para medir los "cinco grandes" y la tríada, y se afirma haber encontrado evidencias confirmatorias de estudios previos de correlación entre ambos grupos de rasgos. Es destacable que en gemelos monocigóticos (gemelos idénticos, comparten ADN idéntico) y gemelos dicigóticos (gemelos no idénticos, no comparten ADN idéntico) se demostró la relevancia de un componente hereditario de moderado a fuerte para el narcisismo y la psicopatía; en cambio, para el maquiavelismo, ligeramente hereditario y con una pronunciada influencia del ambiente compartido ${ }^{22}$. También se concluyó que en el análisis multivariado la correlación existente entre la tríada y los "cinco grandes" es atribuible, en gran medida, a la influencia de los mismos genes ${ }^{23}$.

(2) En cuanto a la influencia hereditaria y el nivel de desarrollo moral, se investigó en una muestra de 154 pares de gemelos monocigóticos y 82 pares de gemelos dicigóticos del mismo sexo (todos entre una edad de 18 y 72 años, de los cuales 372 individuos eran mujeres $)^{24}$. Los datos arrojados revelaron una correlación positiva de los maquiavelistas y los psicópatas con los niveles bajos de desarrollo moral, y también una correlación negativa de los puntajes de los psicópatas en relación con los niveles de alto desarrollo moral. Las diferencias individuales en los bajos niveles de desarrollo moral fueron atribuidas a factores hereditarios y no al ambiente compartido. En cambio, se estableció que los altos niveles de desarrollo moral no se deben a factores genéticos sino a la influencia ambiental. Es menester aclarar que el desarrollo moral se refiere a constructos racionales respecto de estándares éticos, empatía, simpatía, habilidad para pensar acerca de dilemas morales y el poseer convicciones fuertes acerca de lo que se considera moralmente correcto o incorrecto ${ }^{25}$.

En esta investigación se tomó como base referencial la conocida teoría del desarrollo moral de Lorenz Kolberg, que establece tres niveles de desarrollo moral: preconvencional, convencional y posconvencional, y dos estadios correspondientes a cada nivel. Esta teoría propone que conforme una persona se desarrolla biológicamente, asimismo, de manera correlativa y si su desarrollo es saludable, progresa en su capacidad moral de descentrarse (salir paulatinamente del egoísmo natural) y, por consiguiente, de razonar y comportarse con base, primero, en la propia conveniencia y la idea de la reciprocidad, para luego avanzar hacia la solidaridad y pertenencia

22 Cf. Vernon, Philip A.; Vanessa C. Villani; Leanne C. Vickers y Julie Aitken Harris. "A behavioral genetic investigation of the Dark Triad and the Big 5", cit., pp. 451-452.

23 Ibídem, p. 451.

24 Campbell, Jennifer; Julie Aitken Schermer; Vanessa Villani C.; Brenda Nguyen; Leanne Vickers y Philip A. Vernon. "A Behavorial Genetic Study of the Dark Triad of Personality and Moral Development”, cit., p. 133.

25 Ídem. 
a los grupos sociales, la obediencia de la ley y, finalmente, hacia la actuación conforme a principios éticos universales más que conforme a normas, sin que esto último implique su desobediencia (en el nivel avanzado de desarrollo moral, la prioridad es vivir conforme al reconocimiento, defensa y práctica de los derechos humanos como referentes éticos universales $)^{26}$.

(3) En lo tocante al vínculo entre la tríada y los trastornos de personalidad patológicos, la investigación más reciente consignó que los estudios previos sólo habían buscado la correlación con rasgos de personalidad de la población normal, especialmente con los "cinco grandes", por lo cual era necesario abordar la posible correlación entre la tríada y los desórdenes psiquiátricos del grupo B del DSM-V de la American Psychiatric Association (APA) ${ }^{27}$.

Sobre tal presupuesto, el análisis se realizó con una muestra de 661 participantes, elegidos entre estudiantes universitarios y personas convocadas digitalmente a través de la agencia Amazon Mechanical Turk ${ }^{28}$. De entrada, el examen presenta una caracterización del grupo B como individuos de comportamiento impulsivo y errático, que con frecuencia entran en conflictos interpersonales y son proclives al dramatismo, la emocionalidad y el desacierto ${ }^{29}$.

Y en lo referente a la tríada, afirma:

1. Que el alto maquiavelismo tiende a ser de nivel clínico (patológico), carente de principios morales y creyente en la manipulación de las personas como la clave del éxito; quienes lo padecen tienen poco sentido moral, por lo cual incurren en comportamientos sin ética y, así mismo, piensan que "si es necesario elegir entre ser temido y ser amado, es preferible lo primero" 30 . Aunque el maquiavelismo no se correlaciona directamente con el grupo B, al estar traslapado con los demás rasgos de la tríada, se estima que podría contribuir a los trastornos del grupo B, aun cuando esto no está suficientemente claro.

2. El narcisismo se caracteriza por una inflación de la autoestima, sentimientos de grandiosidad y necesidad de admiración por parte de los demás; y se apunta que

26 Sobre el tema, vid., por ejemplo, Habermas, Jürgen. Consciencia moral y acción ..., cit., pp. 121-176.

27 Vossen, Taylor J.; Frederick L. Coolidge; Daniel L. Segal y Jennifer J. Muehlenkamp. “Exploring the Dark Side...", cit.

28 Es un mercado virtual a través del que se puede convocar personas para realizar, por ejemplo, tareas de investigación simples o participación en encuestas, entre muchas más posibilidades de actividades de corte laboral.

29 Vossen, Taylor J.; Frederick L. Coolidge; Daniel L. Segal y Jennifer J. Muehlenkamp. "Exploring the Dark Side...", cit., p. 318 .

30 Ídem. 
existe una relación de continuidad con el narcisismo patológico del grupo B, pero, en tanto el narcisismo subclínico es adaptativo a la vida cotidiana, el patológico entra en conflicto con ésta-es disfuncional.

3. Los individuos psicópatas, por su parte, son definidos como muy impulsivos, sin empatía ni remordimientos, manipuladores, que experimentan baja ansiedad y se orientan a la búsqueda de emociones fuertes; además, a la psicopatía -entre hombres y mujeres- se le considera un fuerte predictor del trastorno antisocial de la personalidad, aunque existe consenso en que ambos padecimientos se traslapan ${ }^{31}$. En los hombres, la psicopatía fue el único predictor significativo de trastorno límite de la personalidad del grupo B (TLP). Para el caso de las mujeres, tanto la psicopatía como el maquiavelismo resultaron predictores significativos de (TLP), pero el maquiavelismo fue el más fuerte.

El último hallazgo sobre esta mayor contribución del maquiavelismo al TLP en las mujeres fue inesperado, y nuevamente puede reflejar una influencia creciente de la cultura sobre las mujeres en la sociedad occidental, y también puede ser expresión de una agresividad creciente que podemos ver en las mujeres con rasgos de TLP ${ }^{32}$.

En algunos trabajos se ha hipotetizado que la psicopatía subclínica se expresa en las mujeres como TLP, mientras que otros defienden que se expresa como trastorno histriónico de la personalidad (THP). Por su lado, el estudio ahora revisado apoya esta última afirmación. Así mismo, que el maquiavelismo tiene una contribución más fuerte que la psicopatía al TLP; además, se encontró al narcisismo como el mayor contribuyente al $\mathrm{THP}^{33}$.

Por último, en general para hombres y mujeres, en relación con el trastorno antisocial de la personalidad del grupo $\mathrm{B}$, entre los principales hallazgos se encontró que

[...] la psicopatía tenía la asociación más fuerte con el trastorno antisocial de la personalidad TAP (efecto fuerte). El maquiavelismo fue la segunda asociación más fuerte (efecto fuerte), y el narcisismo el tercero (efecto medio). Los análisis de regresión lineal para los hombres revelaron que la psicopatía era el único predictor significativo de TAP. Para las mujeres, tanto la psicopatía como el maquiavelismo fueron predictores significativos para TAP, siendo la

31 Ídem.

32 "The latter finding of the contribution of Machiavellianism to BPD in women was unexpected and again may reflect an increasing influence of culture upon women in Western society and may also reflect an increasing aggressiveness we may see in women with BPD features." Ibídem, p. 323 (traducción propia).

33 Ídem. 
psicopatía el predictor más fuerte [...] la contribución significativa del maquiavelismo fue inesperada y requiere más investigación ${ }^{34}$.

En igual sentido, en cuanto al narcisismo, el análisis de regresión lineal reveló, tanto en hombres como mujeres, ser el único predictor significativo de trastorno histriónico de la personalidad (THP) $)^{35}$.

Finalmente, el estudio advierte la limitación de sus resultados puesto que la edad de los individuos de su muestra oscilaba entre los 22 y 30 años. Además, hace mención del agregado a la tríada -en trabajo posterior de uno de los autores originales del concepto- del rasgo sadismo, por lo cual ésta se convierte, como vimos antes, en la tétrada obscura de la personalidad ${ }^{36}$.

De forma complementaria, procede agregar algunas observaciones sobre la "tétrada obscura" hechas por el autor del concepto:

(a) Este grupo puede ubicarse como "constelación de la insensibilidad" (es decir, de la falta de escrúpulos), al que también podrían ser candidatas las personalidades con trastorno límite y la orientada hacia la dominancia social.

(b) Todavía así, no cabe verlas como personalidades clínicamente trastornadas, aun cuando sean agresivas y desleales, a menos que se utilice como criterio la malevolencia social. Pero, en sí mismos, tales rasgos no deberían considerarse patológicos.

(c) Es importante investigar estos "rasgos obscuros", entre otras razones porque la población normal puede presentar, traslapados, tanto rasgos positivos como obscuros de la personalidad.

(d) E igualmente porque muchos individuos con "rasgos obscuros" a menudo se involucran en actividades policiales, militares, políticas, empresariales o de alta influencia social. Personas que llegan a cometer abusos de poder, delitos de cuello blanco y, en general, diversos comportamientos que impactan de modo negativo la vida en sociedad ${ }^{37}$.

Respecto a lo dicho en "b", es oportuno advertir que tal consideración se hace, quizás, porque las personas con tales rasgos resultan funcionalmente "sanas" en una sociedad

34 Ídem (traducción propia).

35 Ídem.

36 Vid. Paulhus, Delroy L. "Toward a Taxonomy of Dark Personalities", cit.

37 Cf. ibídem, pp. 423-424. 
en que la agresividad, la violencia, la manipulación, la mentira, la explotación y el individualismo se han vuelto rasgos normalmente compartidos. En tal circunstancia, el criterio de malevolencia social resulta apropiado porque los individuos con tales rasgos sí producen daño en múltiples esferas de la vida social.

\subsection{Vínculos entre la objetividad y el sentido del comportamiento malevolente}

Tras el recorrido panorámico realizado, es pertinente establecer los vínculos entre las indagaciones empíricas revisadas y las enfocadas en la detección de lo que subyace bajo los rasgos de personalidad expuestos, en particular aquellos proclives a producir daño (la tétrada). En esto cobran especial importancia los últimos dos artículos revisados porque examinan la correlación -digamos, la frontera- entre normalidad y patología o, con mayor precisión, entre lo objetivo y lo no tan evidente, respecto a las posibles motivaciones detrás de las personalidades obscuras.

En efecto, procede decir que los análisis de correlación entre los comportamientos malevolentes ("rasgos obscuros") y los trastornos de la personalidad del grupo B del DSM se conducen de manera objetiva en tanto describen, con cierta puntualidad, enlaces y límites entre las personalidades que, aún de alta nocividad, no pueden considerarse de trato social extraviado y aquellas que, de una u otra manera, tienden a perder piso en el mundo de las relaciones humanas. En otros términos, los criterios objetivos en la investigación de la personalidad se mantienen como tales porque sirven para describir y medir características o "formas de ser" manifiestas de los individuos: sus rasgos. Y es posible correlacionar éstos entre sí o con los de otros individuos que padecen trastornos de personalidad, porque estos últimos también han sido descritos y medidos objetivamente en los manuales internacionales de salud mental, entre los cuales los más reconocidos son: (1) la Clasificación Internacional de Enfermedades (CIE-10) de la Organización Mundial de la Salud y (2) el Manual Diagnóstico y Estadístico de los Trastornos Mentales (DSM) de la Asociación Americana de Psiquiatría ${ }^{38}$.

38 En una encuesta a 5000 psiquiatras de 44 países del mundo, publicada en 2011 , el $70 \%$ dijo guiarse en sus diagnósticos por la CIE-10, en tanto el 23\% reveló que emplea el DSM-IV. "En Europa, estos datos eran incluso más acusados: más del $80 \%$ de unos 2.700 psiquiatras europeos que participaron en la encuesta usaban más la CIE-10, y sólo el 13\% usaba más frecuentemente el DSM-IV." Vid. entrevista a Geoffrey M. Reed, director de la Clasificación de los trastornos mentales y del comportamiento de la Clasificación Internacional de Enfermedades, edición 11 (en proceso de elaboración), Gualberto Buela-Casal. “CIE-11 o DSM-V ¿Cuál debemos utilizar? Entrevista al director de la clasificación de los trastornos mentales y del comportamiento de la CIE-11 (OMS)", 2012 [en línea], disponible en [http:// www.infocop.es/view_article.asp?id=3848]. Por otro lado, en la misma entrevista, Reed afirma: "Según la información que tenemos [existe] una subestimación del grado en que se usa la CIE a nivel global. Ciertamente, el DSM ha sido más usado en la investigación y por lo tanto tiene mayor representación en publicaciones. Sin embargo, encontramos el patrón opuesto en la práctica clínica”. Ibídem. 
Ahora bien: estos referentes internacionales - de salud y enfermedad mental-son documentos elaborados por múltiples equipos de especialistas que han trabajado en la difícil tarea de mantener criterios objetivos en la descripción de los trastornos de personalidad, al punto que puedan emplearse desde cualquier perspectiva teórica de investigación. Incluso, en la elaboración de la quinta edición del DSM (DSM-5) se ha introducido un nuevo enfoque teórico de interpretación y diagnóstico de los trastornos de personalidad alternativo al tradicional, vigente, en ese mismo instrumento ${ }^{39}$.

En relación con ello, vale mencionar que tal óptica representa un importante avance en el abordaje del estudio y la comprensión de los trastornos mencionados debido a que propone estudiarlos de una manera integradora; en la cual se reconoce que los padecimientos se traslapan y, por consiguiente, conviene examinarlos en una perspectiva dimensional más que categórica, como tradicionalmente se ha llevado a cabo a lo largo de las distintas ediciones del DSM y la CIE-10 ${ }^{[40]}$. Respecto a la diferencia entre lo dimensional y lo categórico en relación con los trastornos de personalidad, el DSM-5 dice:

El enfoque diagnóstico utilizado en este manual representa la perspectiva categorial de que los trastornos de la personalidad son síndromes clínicos cualitativamente distintos. [En cambio] Una alternativa al enfoque categórico es la perspectiva dimensional que considera los trastornos de la personalidad como variaciones desadaptativas de los rasgos de la personalidad, que se mezclan imperceptiblemente con la normalidad y entre ellos ${ }^{41}$.

Esta aportación implica un progreso epistémico notable debido a que, por un lado, aunque se conserva el entendimiento de los trastornos como padecimientos cualitativamente distintos uno de otro, como tradicionalmente se ha efectuado en todas las ediciones del DSM (con miras a no interrumpir la práctica clínica basada en esta noción: enfoque categórico), por otro lado, en la perspectiva alterna o dimensional, se concibe a los trastornos en un continuum entre normalidad y patología dentro de la vida en sociedad.

Para nuestro caso, esto último permite apreciar que la malevolencia social que más arriba adjudicamos a los rasgos obscuros de la personalidad (la tétrada) es un criterio

39 Vid. Asociación Americana de Psiquiatría, DSM-5, México, Médica Panamericana, 2014, versión en español. En particular, Sección III: "Modelo alternativo del DSM-5 para los trastornos de la personalidad", pp. 761-781.

40 Los dos documentos internacionales han logrado unificar sus criterios mediante códigos usados para identificar sus respectivas descripciones de cada trastorno, y que son referidos, con reciprocidad, entre uno y otro.

41 Asociación Americana de Psiquiatría, DSM-5..., cit., p. 646 (cursivas mías). 
que ayuda a entender el vínculo imperceptible entre salud y enfermedad dentro de la vida social normal. $\mathrm{O}$, dicho de otra manera, examinar los trastornos de la personalidad como "variaciones desadaptativas" de los rasgos normales -y entremezclados con éstos- significa hacerlo con una perspectiva en que la malevolencia, propia de los rasgos obscuros de la personalidad, se presenta ligada, sutil y progresivamente, a la patología.

De tal forma, el enfoque dimensional introduce cinco dimensiones o rasgos patológicos y los relaciona en calidad de extremos de "los cinco grandes", ya que éstos han sido científicamente validados y replicados en múltiples investigaciones. Con esto es posible reconocer un desarrollo teórico que facilita acercarse a un estudio dinámico de la personalidad, cuando la intención es indagar las motivaciones y el sentido de sus rasgos.

Por su parte, la undécima edición de la CIE, según reporta el director de ella, organizará sus criterios con base en una perspectiva multidisciplinaria ${ }^{42}$, en contraste con la óptica unilateral obtenida sólo desde la psiquiatría; la cual, aunque se maneja con apertura al conocimiento, continúa necesariamente arraigada en la ciencia médica; por lo cual, en no pocas ocasiones, este punto de vista prevalece sobre cualquier otro, como pueden ser: la valoración de la vida, las emociones y sentimientos, con fundamento en contextos morales, comunicativos (a través de la representación lingüística o simbólica, por ejemplo), culturales, históricos, artísticos y, en general, de todos aquellos propios de la interacción humana dentro del mundo de la vida.

En síntesis, se aprecia que la investigación en torno al establecimiento de los criterios de clasificación, descripción y diagnóstico de los trastornos de la personalidad, en los documentos internacionales mencionados, va progresando hacia una perspectiva científica más abierta a la inclusión de diferentes modelos epistemológicos para examinar e interpretar la enfermedad mental.

\subsection{Estudios guiados por el paradigma hermenéutico de investigación}

Hasta aquí resulta pertinente el modelo positivista en la indagación de la personalidad. En esto, el canon objetivo representado -en el caso que nos ocupa- por la capacidad de adaptación social es insuficiente; se precisa de criterios alternos a los objetivos para establecer si la malevolencia de las personalidades obscuras es o no morbosa.

En ello, la tradición de origen europeo en el estudio de la personalidad y el carácter, por medio de la psicología dinámica o psicoanálisis, ha ganado terreno en la indagación de

42 "El Departamento de Salud Mental y Abuso de Sustancias de la oms ya había mostrado su compromiso para que la revisión de los trastornos mentales y conductuales de la CIE contara con una perspectiva multidisciplinar”: Buela-Casal, Gualberto. “CIE-11 o DSM-V ¿Cuál debemos utilizar? Entrevista al director de la clasificación de los trastornos mentales y del comportamiento de la CIE-11 (OMS)", cit. 
las motivaciones ocultas del comportamiento (eminentemente por medio del modelo hermenéutico de investigación), privilegiando en esto el concepto clave de energía, útil para entender y examinar la integración y funcionamiento de la mente humana:

[...] se refiere a fuerzas psíquicas, visibles sólo mediante sus manifestaciones, que tienen cierta intensidad y cierta dirección. Esta energía envuelve, unifica y mantiene unido al individuo dentro de sí mismo así como al individuo en sus relaciones con el mundo exterior. El punto esencial [...] es el concepto dinámico de la conducta humana; es decir, el supuesto de que motivan la conducta fuerzas altamente cargadas, y que la conducta sólo puede comprenderse y preverse conociendo esas fuerzas ${ }^{43}$.

Es importante subrayar que se pueden tener múltiples ideas sobre lo que tendría que entenderse en relación con el origen y procesamiento de la energía mencionada, que bien puede aludir a la energía vital en general -no sólo la libido o energía sexual freudiana- o a la empleada por un individuo para conducirse, deliberada o inconscientemente, en su modo de vida y relación con los demás. Lo fundamental en esta perspectiva es partir de que el individuo emplea energía en cada una de las acciones de su vida y en la motivación de ellas, manejo energético que afecta su forma de sentir, ver y comportarse en el mundo ${ }^{44}$.

En cuanto a esto, es oportuno observar que en la teoría psicoanalítica -entre otrasexiste la diferenciación de temperamento, carácter y personalidad. Temperamento y carácter son considerados partes esenciales de la personalidad. El primero, más ligado a la herencia de las disposiciones innatas de reacción ante los estímulos del ambiente, en particular, la intensidad, el ritmo y los umbrales de las respuestas afectivas; por su parte, el carácter se refiere a la organización dinámica de los patrones de comportamiento que refleja el grado general y el nivel de organización de tales patrones ${ }^{45}$. Así, la personalidad puede considerarse la integración dinámica de todos los patrones de

43 Fromm, Erich. El corazón del hombre. Su potencia para el bien y para el mal, 20. ${ }^{a}$ reimpresión, México, Fondo de Cultura Económica, 2010, p. 33.

44 "Al respecto, es interesante el avance en neurociencia sobre el estudio del sistema nervioso y su fisiología, en particular de las emociones y los sentimientos, donde se ubica al concepto de homeostasis más allá del biológicamente tradicional, reconociendo la importancia de la energía en procesos vitales en general y psíquicos en particular: la esencia de la homeostasis -tanto en los organismos unicelulares como pluricelulares- es la gestión de energía: obtenerla y asignarla a tareas básicas como la reparación, la defensa, el crecimiento, la procreación y el mantenimiento de la descendencia [...] la escala de esta tarea es tan grande que sus efectos pueden comenzar en un nivel inferior de la fisiología y manifestarse en los niveles superiores de esa función, a saber la cognición.” Damassio, Antonio. El extraño orden de las cosas. La vida, los sentimientos y la creación de las culturas, Destino, vol. 287, 2018, p. 29 (cursivas mías).

45 Kernberg, Otto. "Agressivity, narcissism and self-destructiveness in the psychotherapeutic relationship: New developments in the psychology and psychotherapy of severe personality disorders", New Haven, CT: Yale University Press, New Haven and London, 2004, pp. 6-8. 
comportamiento ligados al temperamento, el carácter, el sistema de valores internalizado y la capacidad cognitiva. Así mismo, el inconsciente y la identificación constituyen el sistema de motivación dominante y potencialmente conflictivo de la personalidad ${ }^{46}$.

No obstante tales precisiones, conviene advertir que en la tradición psicoanalítica el estudio del carácter cobra una relevancia fundamental. Y cabe decir que su manejo es similar al que la escuela estadounidense hace de los "rasgos"; de tal forma, lo que en esta última se denomina, por ejemplo, "rasgo narcisista", en la perspectiva de la psicología dinámica se le llama "carácter narcisista". Pero a pesar de la equiparación, el abordaje varía, pues en tanto en el primer caso es de tipo descriptivo, en el segundo se efectúa otro tanto, pero, además, se analizan las motivaciones o dinamismo del carácter.

Sobre tal entendido, es pertinente recordar que en psicoanálisis también se parte de que el carácter es el núcleo de las motivaciones de una persona, pues organiza la energía psíquica que ésta emplea para la consecución de sus fines dominantes ${ }^{47}$. Y esto lo consigue por medio de las pasiones o afanes que forman la base de sus patrones de comportamiento, es decir, la raíz de su carácter específico.

Así pues, a partir de este marco conceptual, comencemos por exponer el estudio del carácter narcisista, uno de los tres rasgos obscuros de la personalidad, ahora desde una perspectiva psicodinámica. Primeramente, procede decir que el narcisismo es concebido como una respuesta evolutiva natural. Dado que en el ser humano el comportamiento de origen instintivo ha cedido lugar al de carácter consciente, el narcisismo, al igual que otros tipos de caracteres, es una compensación a la pérdida. Rigurosamente, representa una respuesta natural para la supervivencia del individuo en su estado consciente de naturaleza ${ }^{48}$.

De tal forma, el narcisismo original es denominado "narcisismo primario", y es inherente a todo ser humano desde su nacimiento, es un recurso adaptativo de supervivencia. En tal sentido, no es concebible un individuo que no busque el autocuidado centrando su atención en sí mismo. Pero este narcisismo de supervivencia, en condiciones de salud mental, no prevalece como centro dinámico del carácter en una persona, sólo le ayuda a sobrevivir. Se le denomina narcisismo óptimo, útil al individuo en su interacción con los demás, le sirve para hacerse valer, pero permite la colaboración. Este narcisismo trasciende al individuo y le permite convivir; "se conserva la energía narcisista, pero se usa en interés de la supervivencia del grupo y no de la supervivencia del individuo" 49 . En el extremo patológico, en cambio, el

46 Ibídem, pp. 7-8.

47 Cf. Fromm, Erich. Anatomía de la destructividad humana, México, Siglo XXI, 1985, p. 255.

48 Cf. Fromm, Erich. El corazón del hombre. Su potencia para el bien y para el mal, cit., p. 37.

49 Ibídem, p. 38. 
narcisismo es máximo, deforma el juicio racional en su forma más peligrosa y obstruye las posibilidades de actuar o adaptarse a la convivencia.

¿Cómo puede reconocerse a la persona narcisista? Hay un tipo que es fácilmente identificable. Es el tipo de individuo que presenta todas las señales de satisfacción de sí mismo; puede advertirse que cuando dice unas palabras triviales cree que está diciendo algo de suma importancia. Por lo general no escucha lo que dicen los demás, ni se interesa realmente. (Si es inteligente, procurará ocultar ese hecho haciendo preguntas y fingiendo parecer interesado.) En muchos casos, la orientación narcisista puede ocultarse detrás de una actitud de modestia y humildad; no es raro, realmente, que la orientación narcisista de un individuo tome su humildad como objeto de su autoadmiración ${ }^{50}$.

El peligro del carácter narcisista estriba en su sensibilidad a toda crítica. Debido a que tiene un marcado desinterés en la opinión y, en general, en la vida de los demás, suele ser refractario a otros puntos de vista. Su reacción emocional a la crítica llega a ser hostil y puede desencadenar un comportamiento iracundo. Esto se entiende por su falta de relación genuina con el mundo, pues "el mundo es él". Si se tiene en cuenta esto, es entendible que cualquier ataque a su auto imagen inflada represente una amenaza a toda su existencia; entonces, siente temor, y éste se encubre y da por resultado una ardiente furia. "Esta furia es sumamente intensa, porque no puede hacer nada para disminuir la amenaza mediante una acción adecuada; sólo la destrucción del crítico - o de uno mismo- puede salvarlo de la amenaza a su seguridad narcisista" 51 . Por otra parte, el carácter narcisista, cuando tiene una posición de poder, suele enfocar sus afanes en transformar la realidad a su forma de ver el mundo; se esfuerza por hacer de éste una extensión de sí mismo ${ }^{52}$.

Dentro de la vida común y corriente, es importante distinguir el narcisismo benigno del narcisismo maligno. El primero está asociado a personas que hacen de su trabajo, o del resultado de un esfuerzo personal, un compromiso para consigo mismas, y aunque la energía empleada en ello es narcisista, se equilibra constantemente por su interés en el proceso del trabajo y en el material con que trabaja (sean labores de carpintería, científicas, artísticas, mecánicas, de ingeniería, etc.); "El mismo hecho

50 Ibídem, p. 36.

51 Ibídem, p. 39.

52 Ídem. Hay más ejemplos en la historia de líderes megalomaníacos que se "curaron" el narcisismo transformando el mundo para ajustarlo a él; estos individuos tienen también que tratar de destruir a todos los críticos, ya que no pueden tolerar la amenaza que constituye para ellos la voz de la cordura. Desde Calígula y Nerón hasta Stalin y Hitler, vemos que su necesidad de encontrar creyentes, de transformar la realidad para que se ajustara a su narcisismo, y de destruir a todos los críticos, es tan intensa y desesperada precisamente porque es un intento para evitar el estallido de la locura. Paradójicamente, el factor de demencia que hay en esos líderes también les hace tener éxito. Les da la certidumbre y el sentirse libres de toda duda, que tan fuertemente impresiona al individuo ordinario. 
de que el trabajo haga necesario relacionarse con la realidad frena constantemente el narcisismo y lo mantiene dentro de límites. Este mecanismo puede explicar por qué encontramos tantos individuos narcisistas que al mismo tiempo son altamente creadores" 53 .

En el narcisismo maligno no hay trabajo ni obra, el objeto que mueve su energía no es la producción de algo, sino es lo que se tiene: su cuerpo, apariencia, riqueza o salud, por ejemplo. Mientras que en el narcisismo benigno el individuo siente que "es grande por lo que hace", en el narcisismo maligno es "grande por lo que tiene". Y como no existe posibilidad de limitar esa energía psíquica por algún proceso de generar una obra, el narcicismo maligno, sin límites, se torna agresivo y xenófobo. "Quien aprendió a hacer cosas no puede menos de reconocer que otros han hecho cosas parecidas de maneras parecidas, aun cuando su narcisismo pueda persuadirle de que su logro es mayor que el de los otros. Quien no ha hecho nada encontrará difícil apreciar los logros de otros, y así se verá obligado a aislarse cada vez más en su esplendor narcisista" ${ }^{54}$.

El síndrome de narcisismo maligno es intermedio entre el trastorno narcisista de la personalidad y el trastorno antisocial de la personalidad propiamente, en el cual existe una total ausencia o deterioro de las funciones del super yo ${ }^{55}$. (El super yo o superego es la censura -o límites normativos-impuesta culturalmente, primero, a través de la figura de los padres y después de la autoridad).

Algo que es menester añadir a lo precedente atañe a las motivaciones del carácter narcisista o de cualquier otro tipo. Es decir, la energía psíquica empleada para la consecución de los fines de un individuo se utiliza de acuerdo con las pasiones o afanes que están en la base o raíz de un carácter específico: en el carácter narcisista, la pasión centrada en la persona misma, su obra o la extensión de ésta; en el carácter sádico, en la pasión por explotar a los demás; en la psicopatía, por el control, la omnipotencia y la destrucción. Digamos que las pasiones se arraigan en el carácter de modo inconsciente y, en el mismo sentido, son modeladas por procesos de identificación.

Distintas condiciones han sido relacionadas con la psicopatía: madres débiles, deprimidas o masoquistas, padres explosivos, inconsistentes o sádicos, alcoholismo y otras adicciones, mudanzas frecuentes, pérdidas y rupturas familiares. La autora considera que en estas circunstancias es casi imposible que se desarrolle con normalidad la confianza en los sentimientos de omnipotencia temprana y en el poder de los otros. Esto podría impulsar al niño a dedicar el resto de su vida a buscar los sentimientos de omnipotencia [...].

53 Cf. ídem.

54 Ídem.

55 Kernberg, Otto. “Agressivity, narcissism and self-destructiveness...”, cit., p. 20 (traducción propia). 
Otro rasgo de la experiencia del self [el yo] en el paciente psicopático que merece atención es la envidia primitiva, el deseo de destruir lo que uno más desea $[. .$.$] . Aunque la gente antisocial raramente reconoce la envidia, muchos$ de sus comportamientos la demuestran: "Uno probablemente no puede crecer incapaz de amar sin saber que hay algo ahí fuera que otra gente disfruta y de lo que uno carece”. La devaluación y [el] desprecio de cualquier manifestación de ternura en la vida humana caracteriza[n] a la gente psicopática; algunos psicópatas psicóticos matan precisamente lo que los atrae ${ }^{56}$.

Pero las pasiones no se presentan solas, sino como síndromes. En el caso que nos ocupa, como síndromes "contrarios a la vida": el sadomasoquismo, la destructividad, la voracidad, el narcisismo, van juntos y tienen una misma orientación básica. En el caso opuesto están el amor, la solidaridad, la justicia y la razón: "síndrome a favor de la vida" 57 . En las personas normales -según el enfoque psicodinámicotales síndromes se comparten, por lo que nadie podría verse totalmente gobernado por pasiones en contra de la vida o totalmente a favor de ésta, sin presentar contradicciones. El núcleo de esto es que tales pasiones, expresadas como síndromes, conforman el carácter de las personas; esto es, impulsan, motivan la dirección en que se ejerce la energía psíquica orientada a fines.

De tal forma, en lo que corresponde al carácter sádico (o "sadismo de la tétrada"), encontramos que su motivación básica es la explotación, la pasión de poder absoluto e irrestricto sobre un ser vivo, no importa si es humano o no. El humillar y menoscabar a otros, mandar sobre sus vidas, es el centro de su dinámica, y aun cuando en algunos casos pudiera parecer que alguien -en posición sumisa- necesita ser guiado para su beneficio, esto no basta ni justifica el proceder de quien posee un carácter o rasgo sádico. De ahí su malevolencia y ubicación negativa.

El sadismo es una solución al problema de vivir sin productividad y alegría; es transformación de impotencia en omnipotencia: la religión de los lisiados psíquicos; necesita el dominio de otro, aunque desprecia y domina a indefensos que no pueden enfrentarlo. En realidad, el carácter sádico es complementario con el masoquista debido a la díada dominio-sumisión; por esto, con mayor propiedad, se le reconoce como carácter sadomasoquista; no obstante, también se le atribuye, desde un punto de vista político, el apelativo de "carácter autoritario" 58.

Otro elemento del síndrome es la sumisión y cobardía del sádico. Puede parecer una contradicción que el sádico sea sumiso, pero no lo es... dinámicamente

56 De Celis Sierra, Mónica. "Personalidades psicopáticas (antisociales) (McWilliams, N. Diagnóstico psicoanalítico. Comprendiendo la estructura de la personalidad en el proceso clínico)”, en Aperturas Psicoanalíticas. Revista Internacional de Psicoanálisis, n. ${ }^{\circ}$ 047, 2014, pp. 4-6 (cursivas mías).

57 Cf. Fromm, Erich. Anatomía de la destructividad humana ..., cit., p. 258.

58 Cf. ibídem, pp. 292, 294. 
hablando es necesario que así sea. Es sádico porque se siente impotente, sin vida ni poder. Compensa este defecto teniendo poder sobre otros [...] podrá matar o torturar, pero no deja por eso de ser una persona sin amor aislada y asustada, que necesita un poder superior al que someterse. Para los que estaban un escalón más debajo de Hitler, el poder máximo era el Führer; para el propio Hitler, era el Destino, las leyes de la Evolución ${ }^{59}$.

Por su lado, habría que considerar qué tanto se puede asociar la "constelación de la insensibilidad" -los rasgos de la tétrada-con el "carácter necrófilo" descrito en la línea psicodinámica aquí seguida. Esto, porque la clasificación de la tétrada proviene de estudios empíricos que han asumido terminología proveniente básicamente del psicoanálisis. Y, como puede advertirse, los criterios psicodinámicos expuestos tienen mucha fuerza explicativa, aun sin que se haya abundado en análisis especialmente relacionados con el maquiavelismo o, incluso, la psicopatía -acerca de los cuales se requiere mayor trabajo psicoanalítico.

Así, en el carácter necrófilo predomina "la atracción apasionada por todo lo muerto, corrompido, pútrido y enfermizo; es la pasión de transformar lo viviente en algo no vivo, de destruir por destruir, y el interés exclusivo por todo lo puramente mecánico. Es la pasión de destrozar las estructuras vivas"60.

Al respecto, en el análisis se exploran los sueños y las vidas de personajes con tendencias hacia la necrofilia, es decir: la inclinación a interesarse en objetos sin vida, materia putrefacta, máquinas, muerte y enfermedades, sobre lo cual la animación y excitación se apodera de los individuos a la mención de estos aspectos. Su interés en leer obituarios, asistir a salas de velación y cementerios; la forma de comportarse en una conversación - que puede ser rígida y fría-, la glorificación de la guerra, el aniquilamiento de la cultura, el odio a las mujeres, el gusto por la velocidad y las máquinas. En síntesis, un conjunto fuertemente simbólico de alejamiento de aquello que representa generación y sostén de la vida.

La muerte ya no se expresa simbólicamente por heces y cadáveres malolientes. Sus símbolos son ahora máquinas limpias y brillantes; no atraen a los hombres las deposiciones olorosas sino las estructuras de aluminio y vidrio. Pero la realidad que oculta esta fachada antiséptica se hace cada vez más visible. El hombre, en nombre del progreso, está transformando el mundo en un lugar pestilente y envenenado [...] los fenómenos que despiertan tanta indignación -drogadicción, crimen, decadencia cultural y espiritual, desprecio de los valores éticos genuinos- están relacionados todos con la creciente atracción de la muerte y la suciedad. ¿Cómo puede uno esperar que los jóvenes, los pobres 
y los que no tienen esperanza no se sientan atraídos por la decadencia cuando la promueven los que dirigen el curso de la sociedad contemporánea ${ }^{61}$

No sin razón, la explicación psicodinámica advierte la orientación de la humanidad hacia sociedades que organizan la vida en torno al dinero, la violencia y la banalidad. El que seamos gobernados, principalmente, por personalidades apasionadas en la lucha y conquista del poder por el poder mismo nos ubica dentro de un contexto de normalidad enfermiza en que lo patológico se llega a admirar y genera prestigio. ¿Con qué criterios, objetivos o no, habríamos de medir o dar cuenta de los integrantes de una sociedad así y afirmar que gozan de salud?

Ciertamente, para resolver tal interrogante, ayuda mucho el criterio de los síndromes "a favor de la vida" y "en contra de la vida", propuesto desde esta orientación psicoanalítica, el cual va más allá de criterios objetivos de uso limitado y cuestionable, como el del comportamiento adaptativo. Esta clasificación de los síndromes, en apariencia más ética que científica, no resulta descabellada si se toma en cuenta el objeto y razón de la existencia humana y toda su producción, incluida, por supuesto, la que se refiere a la tarea de la ciencia: el hacer que la vida en el planeta florezca en todo su esplendor y posibilidades, por mediación del potencial que da la consciencia y espiritualidad propias de nuestra especie. En tal sentido, el humanismo, la ética y el saber científico son inseparables.

\section{CONCLUSIONES}

Primera. La insensibilidad, inseparable - centro- de algunos trastornos de la personalidad puede ser un problema adaptativo cuando los rasgos están en el extremo patológico del continuum salud-enfermedad; pero si no es así, como en el caso de la tétrada obscura de la personalidad, los rasgos malevolentes resultan perfectamente funcionales dentro de un entorno en que abundan las acciones de desapego y desinterés por los demás o falta de empatía; incluso, engañosamente llegan a reconocerse como "rasgos evolutivamente adaptativos". Piénsese, por ejemplo, en el despliegue de agresividad, violencia, manipulación, fingimiento y explotación de parte ciertos individuos -no pocos- que ejercen actividades empresariales, políticas o de liderazgo ligado a la fe, para quienes lo importante es la influencia y los beneficios obtenidos por ellos de las "masas" de consumidores, ciudadanos o creyentes.

Segunda. Si la ausencia de empatía, el desapego e interés por otros no representa un problema de adaptación, como en el caso de la tétrada, observamos que desaparece, objetivamente hablando, la posibilidad de considerarla mórbida, ya que la adaptabilidad a la vida social es el criterio base para definir si hay o no enfermedad.

61 Ibídem, pp. 348-349. 
Tercera. Sin embargo, ir contra la supervivencia de la especie no puede ser tomado en serio como rasgo adaptativo, pues ocasionar daño a otros por medio de comportamientos vinculados al abuso de poder, acumulación de riqueza, generación de desigualdad nociva a la condición humana y deterioro del ambiente, es decir, de un mundo con pobreza multidimensional -y riesgoso para la vida de otras especiesde ningún modo favorece la evolución de la humanidad y del planeta.

Cuarta. Bajo el criterio de los síndromes "a favor" o "en contra" de la vida, los rasgos o personalidades malevolentes no pueden ser considerados saludables en una normalidad que los incluye como si lo fueran. La malignidad u obscuridad de tales formas de ser y obrar necesariamente son reconocibles como patológicas, aun cuando sus portadores se muevan en el mundo de manera muy adaptada y con mucha capacidad organizativa de grupos y sociedades. El centro de la atención, entonces, se enfoca hacia el tipo de sociedad y mundo en el que podemos vivir y pervivir con dignidad.

\section{REFERENCIAS}

American Psychiatric Association. Guía de consulta de los criterios diagnósticos del $D S M-V$, Chicago, American Psychiatric Publishing, A division of American Psychiatric Association, Washington, D. C.-London, England, 2014.

Asociación Americana de Psiquiatría. DSM-5, México, Médica Panamericana, 2014.

Basarab, Nicolescu. La transdisciplinariedad. Manifiesto, Ed. Du Rocher, 1999.

Belardineli, Sergio. La teoría consensual de Jürgen Habermas, Anuario filosófico, 1991,p. 117 [en línea], disponible en [https://dadun.unav.edu/bitstream/10171/2322/ 1/02.\%20Sergio\%20Belardinelli.\%20La\%20Teor\%C3\%ADa\%20Consensual\%20 de\%20La\%20Verdad\%20de\%20J\%C3\%BCrgen\%20Habermas.pdf].

Buela-Casal, Gualberto. “CIE-11 o DSM-V ¿Cuál debemos utilizar? Entrevista al director de la clasificación de los trastornos mentales y del comportamiento de la CIE-11 (OMS)", 2012 [en línea], disponible en [http://www.infocop.es/view_article.asp?id=3848].

Campbell, Jennifer; Aitken Schermer, Julie; Villani C., Vanessa; Nguyen, Brenda; Vickers, Leanne y Vernon, Philip. "A Behavorial Genetic Study of the Dark Triad of Personality and Moral Development", en Twin Research and Human Genetics, vol. 12, n. $^{\circ} 2,2009$.

Celis Sierra, Mónica de. “Personalidades psicopáticas (antisociales) (McWilliams, N. Diagnóstico psicoanalítico. Comprendiendo la estructura de la personalidad en el proceso clínico)", Aperturas Psicoanalíticas. Revista Internacional de Psicoanálisis, n. ${ }^{\circ} 047,2014$. 
Damassio, Antonio. El extraño orden de las cosas. La vida, los sentimientos y la creación de las culturas, Destino, vol. 287, 2018.

Enríquez Rubio Hernández, Herlinda. Investigación científica en el derecho y disciplinas afines. Un proceso epistémico-metodológico riguroso, México, Porrúa, 2019.

Enríquez Rubio Hernández, Herlinda y Hernández Cuevas, Maximiliano. Consideraciones epistémico-metodológicas sobre la investigación de la realidad jurídicosocial, México, Instituto de Investigaciones Jurídicas de la Universidad Autónoma de Chiapas, 2014 [en línea], disponible en [www.iij-unach.mx].

Fromm, Erich. Anatomía de la destructividad humana, México, Siglo XXI, 1985.

Fromm, Erich. El corazón del hombre. Su potencia para el bien y para el mal, 20. a reimpresión, México, Fondo de Cultura Económica, 2010.

Habermas, Jürgen. Consciencia moral y acción comunicativa, Madrid, Trotta, 2008.

Habermas, Jürgen. La ética del discurso y la cuestión de la verdad, 3. impresión, México, Paidós, 2010.

Habermas, Jürgen. Teoría de la acción comunicativa, México, Taurus, 2002.

Hernández Cuevas, Maximiliano. La investigación argumentada. Bases del discurso en la ciencia y en el derecho, México, Porrúa, 2017.

Kernberg, Otto. "Agressivity, narcissism and self-destructiveness in the psychotherapeutic relationship: New developments in the psychology and psychotherapy of severe personality disorders", New Haven, CT: Yale University Press, New Haven and London, 2004.

Morin, Edgar. El método III. El conocimiento del conocimiento, Madrid, Cátedra, 1999.

Osorio García, Sergio Néstor. "El pensamiento complejo y la transdisciplinariedad: fenómenos emergentes de una nueva racionalidad", Rev. Fac. Cienc. Econ., vol. Xx n. ${ }^{\circ} 1$, Universidad Militar Nueva Granada, junio de 2012.

Paulhus, Delroy L. “Toward a Taxonomy of Dark Personalities”, Current Directions in Psychological Science, vol. 23 (6), 2014 [en línea], disponible en [cdp.sagepub.com].

Piaget, Jean y García, Rolando. Psicogénesis e historia de la ciencia, 11. a ed., México, Siglo XXI, 2008. 
Tarski, Alfred. La concepción semántica de la verdad y los fundamentos de la semántica, 1966 [en línea], disponible en [http://serbal.pntic.mec.es/ cmunoz11/tarski.pdf].

Tarski,Alfred. "Verdad y demostración”, Disputatio. Philosophical Research Bulletin, vol. 4, n. ${ }^{\circ}$ 5, dic. 2015 [en línea], disponible en [www.disputatio.eu]. 